\title{
White radish and swine scapular cartilage models for auricular framework carving training
}

\section{Kun Hwang}

Department of Plastic Surgery, Inha University School of Medicine, Incheon, Korea

\begin{abstract}
Background: The aim of this study is to develop a two-stage training module using radish and swine scapular cartilage for carving ear cartilage.

Methods: In the first stage, white radish was cut in 3-6 mm thick slices. The ear cartilage framework was carved using a graver and the helix and antihelix were fixed with pins. In the second stage, swine scapular cartilage was obtained. The thickness varied 3-6 mm. The ear cartilage framework was made. And triangular fossa and scaphoid fossa were carved with graver. A curvilinear cartilage for helix was assembled to the framework by pin fixing. Six participants were recruited for an ear reconstruction training workshop and figures of the cartilage framework were provided. Participants were asked answer the pre-workshop questionnaire and post-workshop questionnaire on a Likert scale to rate their satisfaction with the outcome.

Results: On the pre-workshop questionnaire, participants indicated that they did not have sufficient knowledge and skill for fabricating the ear cartilage framework $(1.5 \pm 0.5$ using white radish; $1.3 \pm 0.5$ using swine scapular cartilage). On the post-workshop questionnaire, participants responded that they had learned useful knowledge from this workshop, reflecting a significant improvement ( $3.8 \pm 1.0$ using white radish; $4.0 \pm 1.1$ using swine scapular cartilage). They also indicated that they had become somewhat confident in this skill $(4.2 \pm 0.8$ using white radish; $4.3 \pm 0.5$ using swine scapular cartilage. The participants generally found the workshop satisfactory (practically helpful, $4.7 \pm 0.5$; knowledge improved, $4.8 \pm 0.4$; satisfied with course, $4.5 \pm 0.5$; would recommend to others, $4.8 \pm 0.4$ ).

Conclusion: This model can be useful for ear reconstruction training for medical personnel.
\end{abstract}

Keywords: Congenital microtia / Ear auricle / Education / Models, anatomic / Simulation

\section{INTRODUCTION}

The most common clinical technique for ear reconstruction is surgical reconstruction with autologous tissue. This procedure requires the surgeon to have a high level of manual dexterity and

Correspondence: Kun Hwang

Department of Plastic Surgery, Inha University School of Medicine, 27 Inhang-ro, Jung-gu, Incheon 22332, Korea

E-mail: jokerhg@inha.ac.kr

This study was supported by a grant from National Research Foundation of Korea (NRF-2020R1I1A2054761).

The author is grateful to Hun Kim, PhD., Department of Plastic Surgery, Inha University School of Medicine, for making tables.

Received July 3, 2020 / Revised July 23, 2020 / Accepted August 12, 2020 artistic technique when carving and sculpting the harvested costal cartilage. The aesthetic outcomes of these surgical procedures are highly dependent on the experience of the surgeon [1]. Some models for training have been developed using soaps and alloplastic materials [2-4]. Recently, ox scapular cartilage was introduced to simulate carving ear cartilage [5]. In our previous study, swine scapular cartilage was obtained for a nasal septal cartilage model. The thickness was 3 to $6 \mathrm{~mm}$, and the size varied depending on the animal's weight [6]. The aim of this study is to develop a two-stage training module using a radish and swine scapular cartilage for carving ear cartilage. 


\section{METHODS}

\section{Stage 1: White radish model}

A white radish was cut in 3- to 6-mm-thick slices. The ear cartilage framework was carved using a graver. The helix and antihelix were fixed with pins (Fig. 1).

\section{Stage 2: Swine cartilage model}

Swine scapular cartilage was obtained. The thickness varied from 3 to $6 \mathrm{~mm}$ (Fig. 2A). The ear cartilage framework was made (Fig. 2B). And triangular fossa and scaphoid fossa were carved with graver (Fig. 2C). A curvilinear cartilage for helix (Fig. 2D) was assembled to the framework by pin fixing (Fig. 2E).

\section{Participants}

Six participants (three plastic surgery residents and three graduate

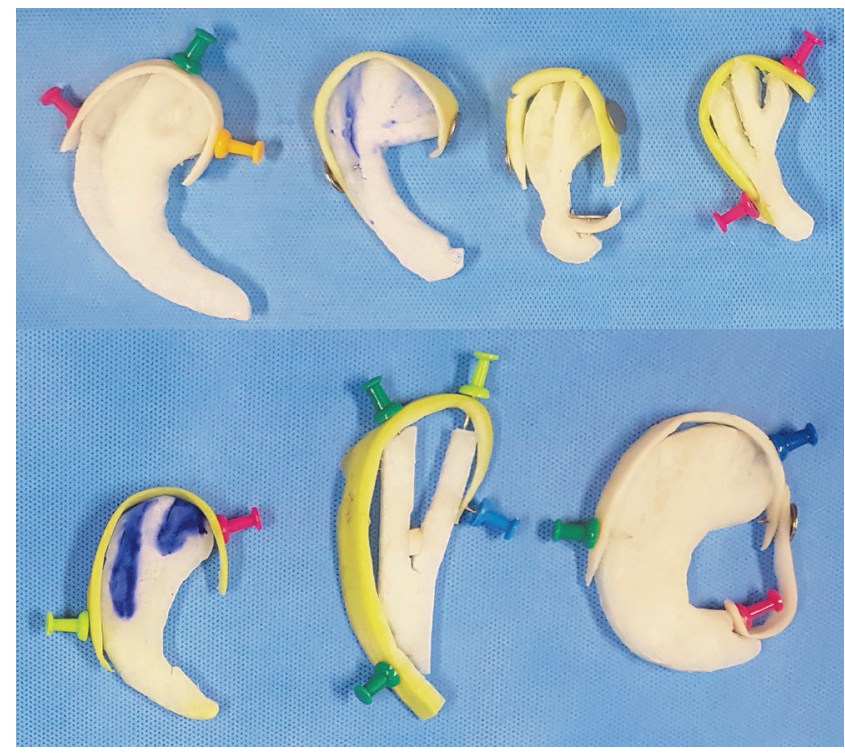

Fig. 1. Auricular cartilage frameworks using white radish.
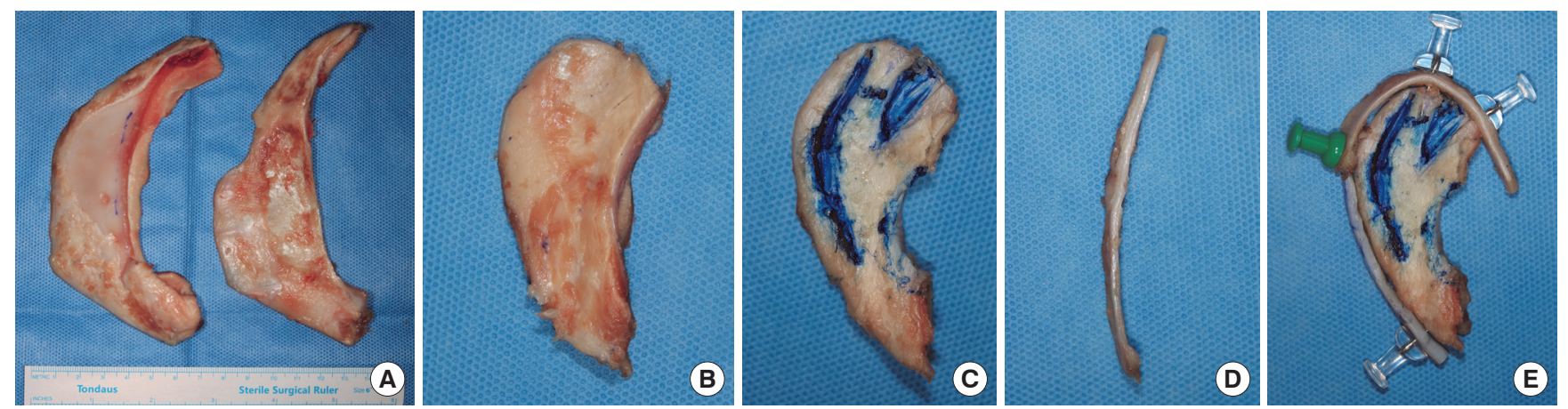

Fig. 2. Auricular cartilage framework using swine scapular cartilage. (A) Harvested swine scapular cartilage. The thickness varied from 3 to 6 $\mathrm{mm}$. (B) The ear cartilage framework was made. (C) Triangular fossa and scaphoid fossa were carved with graver. (D) A curvilinear cartilage for helical rim. (E) Framework assembled by pin fixing. 
Table 1. Answers to ear reconstruction training

\begin{tabular}{lrrrr}
\hline \multirow{2}{*}{ Answer } & \multicolumn{4}{c}{ Model } \\
\cline { 2 - 5 } & White radish & $p$-value & Swine cartilage & $p$-value \\
\hline Pre-workshop & & & & \\
$\quad$ Knowledge, skill & $1.5 \pm 0.5$ & & $1.3 \pm 0.5$ & \\
Post-workshop & & & & \\
Knowledge & $3.8 \pm 1.0$ & 0.001 & $4.0 \pm 1.1$ & $<0.001$ \\
Confidence of skill & $4.2 \pm 0.8$ & $<0.001$ & $4.3 \pm 0.5$ & $<0.001$ \\
Practically helpful & $4.7 \pm 0.5$ & & $4.5 \pm 0.8$ & \\
\hline
\end{tabular}

Values are presented as mean \pm SD. Participants were asked to rate their satisfaction with the outcome of a Likert scale (5: definitely yes, 4: yes, 3: moderate, 2: no, 1: definitely no).

\section{DISCUSSION}

Microtia reconstruction is a challenging procedure for trainees. The most complex part is learning how to fabricate a framework from costal cartilage. Some models have been developed for training using soaps, alloplastic materials, or ox scapular cartilage. Murabit et al. [2] used silicone costal cartilage models. Thadani and Ladani [3] wrote that a silicone dental impression material yielded a consistency and texture that were comparable to those of real costal cartilage. Erdogan et al. [4] used a polyvinyl chloride plastic eraser since its texture was similar to that of human costal cartilage. Agrawal [5] used ox scapular cartilage because its consistency, flexibility, and cutting experience almost matched that of human costal cartilage. Trainees also might use human rib cartilage from cadavers, however, fixed cadaveric cartilages are not easily available and too hard to carve because they are dehydrated and usually from old aged donors. In our previous study, swine scapular cartilage was obtained for a nasal septal cartilage model. The thickness was 3 to $6 \mathrm{~mm}$, and the size varied depending on the animal's weight [6].

Our model can be simply made using easily obtained white radish and swine scapular cartilage, which are available at grocery stores and butcher shops, respectively. Using a burin and pins, trainees could feel the tactile sensation of carving a radish or cartilage. Radish model is easy to carve, so the trainees can express their creativity. However, radish does not have similar consistency with human rib cartilage. Swine scapular cartilage has similar consistency with human rib cartilage. After becoming skilled with radish model, swine scapular cartilage can also be carved easily for desired framework. Through a training workshop using our model, three residents and three graduate students who had never previously performed ear reconstruction obtained a full understanding of the procedures. They all became somewhat confident in their knowledge and skill after the workshop.

The present study is a study based on one department and has
Table 2. Satisfaction to the workshop

\begin{tabular}{lc}
\hline Question & Score \\
\hline Practically helpful & $4.7 \pm 0.5$ \\
Knowledge improved & $4.8 \pm 0.4$ \\
Satisfied with this course & $4.5 \pm 0.5$ \\
Will recommend this course to others & $4.8 \pm 0.4$ \\
\hline
\end{tabular}

Values are presented as mean $\pm S D$. Participants were asked to rate their satisfaction with the outcome of a Likert scale (5: definitely yes, 4: yes, 3: moderate, 2: no, 1: definitely no).

limited number of participants. Further study would be followed based on larger workshop for the trainee at congresses. With larger numbers of participants, better assessment tool other than pre- and post-workshop self-report could be applied. This model can be useful for ear reconstruction training for medical personnel.

\section{NOTES}

\section{Conflict of interest}

No potential conflict of interest relevant to this article was reported.

\section{Ethical approval}

The study was approved a special exemption by the Institutional Review Board of Inha University School of Medicine (exemption No. 200708-1A).

\section{ORCID}

Kun Hwang https://orcid.org/0000-0002-1994-2538

\section{Supplementary material}

Supplementary Table S1. Pre-workshop questionnaire.

Supplemental data can be found at: https://doi.org/10.7181/ acfs.2020.00297.S1.

Supplementary Table S2. Post-workshop questionnaire.

Supplemental data can be found at: https://doi.org/10.7181/ acfs.2020.00297.S2.

\section{REFERENCES}

1. Mussi E, Furferi R, Volpe Y, Facchini F, McGreevy KS, Uccheddu F. Ear reconstruction simulation: from handcrafting to 3D printing. Bioengineering (Basel) 2019;6:14.

2. Murabit A, Anzarut A, Kasrai L, Fisher D, Wilkes G. Teaching ear reconstruction using an alloplastic carving model. J Craniofac Surg 2010;21:1719-21.

3. Thadani SM, Ladani PS. A new method for training of ear 
framework creation by silicon dental impression material. Indian J Plast Surg 2012;45:134-7.

4. Erdogan B, Morioka D, Hamada T, Kusano T, Win KM. Use of a plastic eraser for ear reconstruction training. Indian J Plast Surg 2018;51:66-9.

5. Agrawal K. Bovine cartilage: a near perfect training tool for carving ear cartilage framework. Cleft Palate Craniofac J 2015; 52:758-60.
6. Hwang SH, Hwang K. Obtaining a model of nasal septal cartilage from swine scapular cartilage. J Craniofac Surg 2012;23: 1222-3.

7. Brent B. Ear reconstruction with an expansile framework of autogenous rib cartilage. Plast Reconstr Surg 1974;53:619-28.

8. Brent B. The correction of mi-rotia with autogenous cartilage grafts: I. The classic deformity. Plast Reconstr Surg 1980;66:112. 
Supplementary Table S1. Pre-workshop questionnaire

- Title: Ear reconstruction training workshop

- Date:

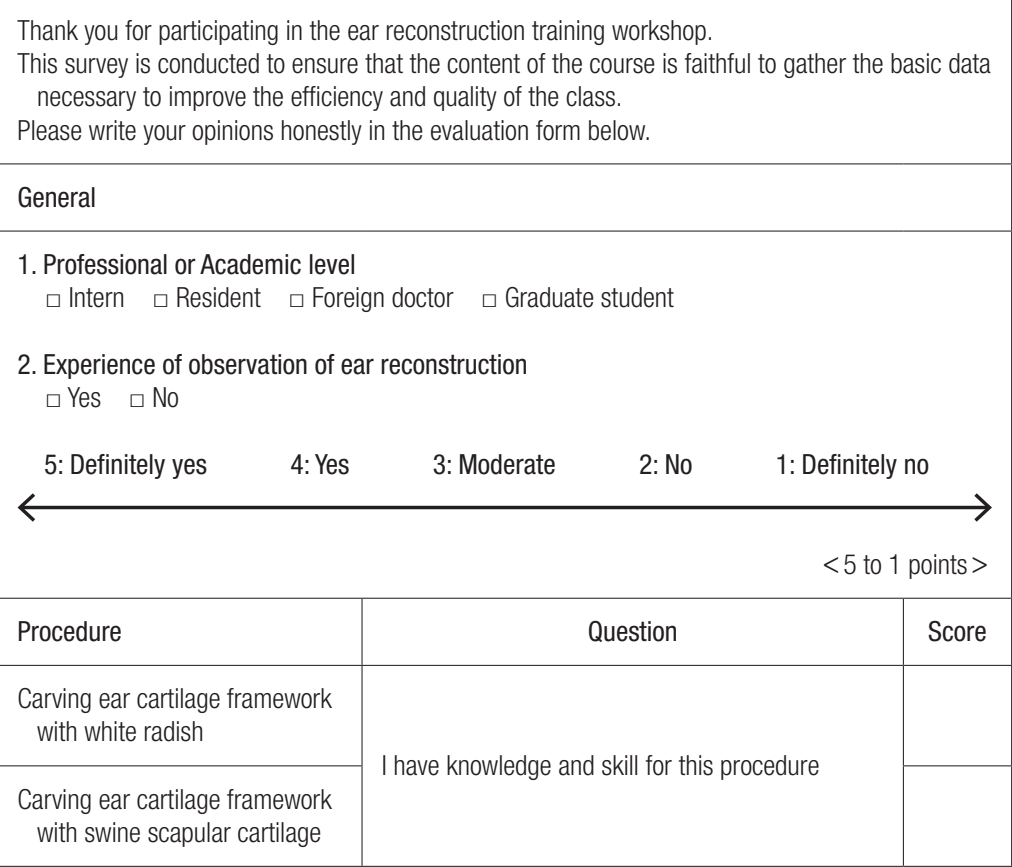


Supplementary Table S2. Post-workshop questionnaire

- Title: Ear reconstruction training workshop

- Date:

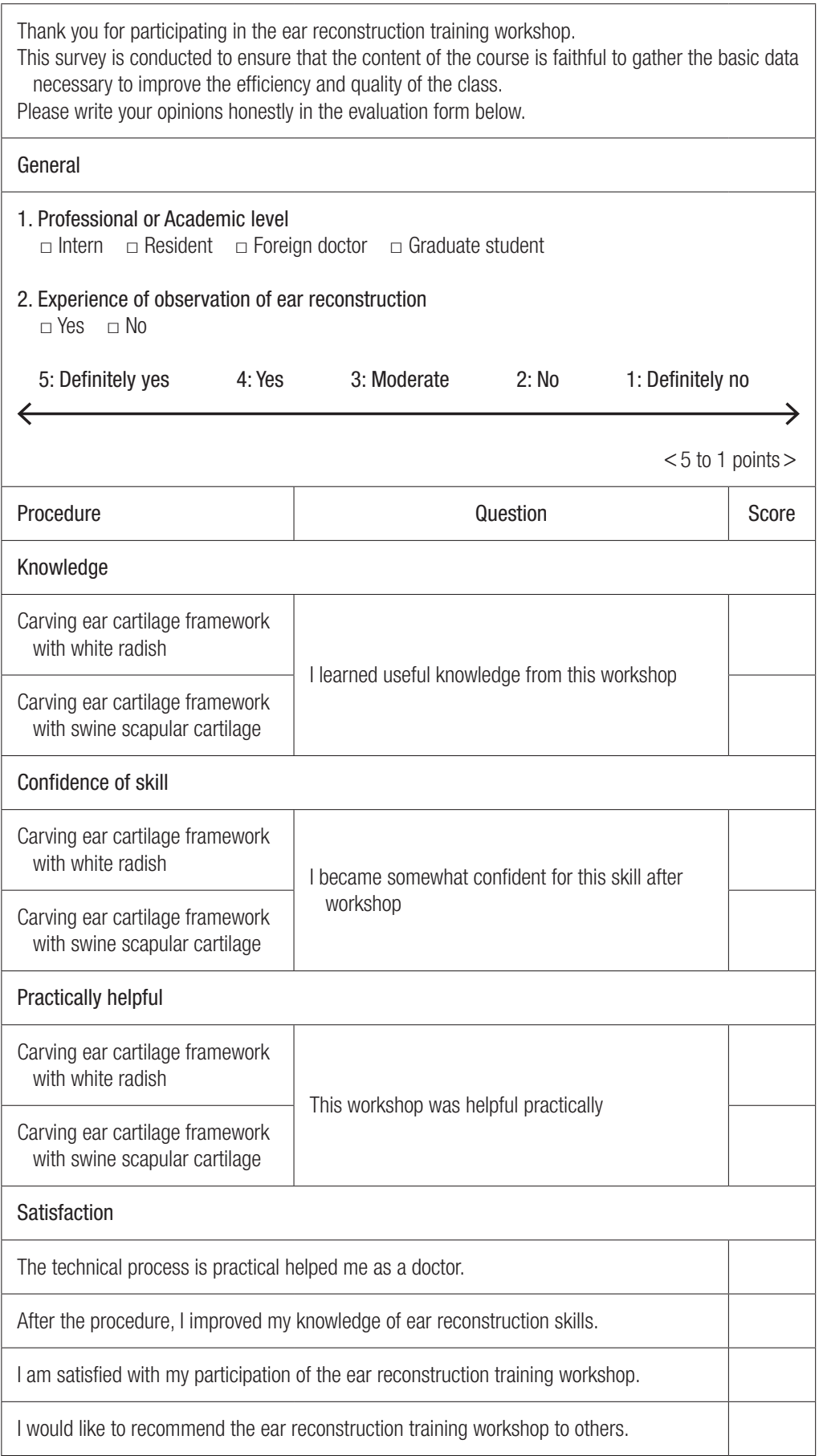

